OPEN ACCESS

Edited by:

Xing Tian,

New York University Shanghai, China

Reviewed by:

Sebastian Halder, University of Oslo, Norway

Erwei Yin,

China Astronaut Research and

Training Center, China

Jing Jin,

East China University of Science and

Technology, China

${ }^{*}$ Correspondence:

Peng $X u$

xupeng@uestc.edu.cn

Received: 17 August 2018 Accepted: 11 December 2018 Published: 07 January 2019

Citation:

Li F, Yi C, Jiang Y, Liao Y, Si Y, Dai J,

Yao D, Zhang $Y$ and $X u P$ (2019) Different Contexts in the

Oddball Paradigm Induce Distinct

Brain Networks in Generating

the $P 300$.

Front. Hum. Neurosci. 12:520.

doi: 10.3389/fnhum.2018.00520

\section{Different Contexts in the Oddball Paradigm Induce Distinct Brain Networks in Generating the P300}

\author{
Fali $\mathrm{Li}^{1,2}$, Chanlin $\mathrm{Yi}^{1,2}$, Yuanling Jiang ${ }^{1,2}$, Yuanyuan Liao ${ }^{1,2}$, Yajing $\mathrm{Si}^{1,2}$, Jing Dai ${ }^{1}$, \\ Dezhong Yao ${ }^{1,2}$, Yangsong Zhang ${ }^{1,2,3}$ and Peng $X u^{1,2 *}$
}

${ }^{1}$ MOE Key Lab for Neuroinformation, The Clinical Hospital of Chengdu Brain Science Institute, University of Electronic Science and Technology of China, Chengdu, China, ${ }^{2}$ Center for Information in Medicine, School of Life Science and Technology, University of Electronic Science and Technology of China, Chengdu, China, ${ }^{3}$ School of Computer Science and Technology, Southwest University of Science and Technology, Mianyang, China

Despite the P300 event-related potential (ERP) differences between distinct stimulus sequences, the effect of stimulus sequence on the brain network is still left unveiled. To uncover the corresponding effect of stimulus sequence, we thus investigated the differences of functional brain networks, when a target $(T)$ or standard $(S)$ stimulus was presented preceding another $T$ as background context. Results of this study demonstrated that, when an S was first presented preceding a T (i.e., ST sequence), the P300 experiencing large amplitude was evoked by the T, along with strong network architecture. In contrast, if a T was presented in advance [i.e., target-to-target (TT) sequence], decreased P300 amplitude and attenuated network efficiency were demonstrated. Additionally, decreased activations in regions, such as inferior frontal gyrus and superior frontal gyrus were also revealed in $\Pi$ sequence. Particularly, the effect of stimulus sequence on P300 network could be quantitatively measured by brain network properties, the increase in network efficiency corresponded to large P300 amplitude evoked in P300 task.

Keywords: background context, brain network, P300, response variances, oddball paradigm

\section{INTRODUCTION}

In the oddball paradigm, two types of stimuli, i.e., target (T) and standard (S), are randomly presented to subjects, the presentation of T can evoke a P300 event-related potential (ERP; Squires et al., 1975; Duncan-Johnson and Donchin, 1977). To evoke a clear P300, subjects are required to only respond to $\mathrm{T}$ in a requested manner (e.g., counting the number or pressing a button) while omitting $S$ in the active paradigm; while in the passive paradigm, subjects would only concentrate on stimuli without responses (Pokorny et al., 2013; Risetti et al., 2013). The P300 shows a largest positive peak at approximately $300 \mathrm{~ms}$ after stimulus onsets, and is found prominently over parietal region (Polich, 2007). The P300 can be used as a physiological biomarker to evaluate brain potential of information processing (Rutiku et al., 2015; Turetsky et al., 2015), and is widely used in clinical diagnosis (Howe et al., 2014; Turetsky et al., 2015; Li et al., 2018b), cognitive neuroscience (van Dinteren et al., 2014; Wang et al., 2015), and brain-computer interface (BCI; Farwell and Donchin, 1988; Yin et al., 2016). A hybrid BCI system combining P300 and steady-state visual evoked potential acquired an online classification accuracy of $93.85 \%$ with information transfer rate of $56.44 \mathrm{bit} / \mathrm{min}$ in only a single trial (Yin et al., 2013), which also achieved the wheelchair control (Li et al., 2013) and detection of awareness in patients with disorders of consciousness (Pan et al., 2014). 
The effect of stimulus sequence on P300 has been investigated since 1976 (Squires et al., 1976; Verleger, 1987). A large P300 amplitude can be evoked, if identical stimuli (e.g., standard) are consecutively presented and then terminate with a different stimulus (e.g., target; Squires et al., 1976); an increase of the number of $\mathrm{S}$ stimulus before a $\mathrm{T}$ corresponds to the increase of P300 amplitude evoked by the $\mathrm{T}$ stimulus (Polich and Bondurant, 1997). Meanwhile, stimulus sequence interacts with interstimulus interval (ISI) and target probability. Small target probability produces a sequence that includes a series of $S$ stimuli and a $\mathrm{T}$ stimulus, which evokes a clear P300. In contrast, the relatively short ISI consumes more brain resources, due to the frequent presentations of stimulus events, small P300 amplitude will be evoked (Polich, 1990).

Particularly, manipulation of global (especially local) $\mathrm{T}$ probability produces the effect of target-to-target interval (TTI) on P300. TTI determines how quickly brain resources can be redirected to process target information (Pashler, 1994); shorter interval produces much smaller P300 than longer interval (Steiner et al., 2013). The positive relationships between TTI and hemodynamic activity in multiple brain regions, such as anterior cingulate, might index that TTI modulates the brain activity related to target in distinct network structures by updating of working memory processes (Stevens et al., 2005).

Time-frequency analysis is of great importance to assess brain activity in ERPs (Busch et al., 2006; Bernat et al., 2007). The generation of P300 is attributed to a series of procedures that include stimuli perception, information integrating, decision processing, and neuronal response ( $\mathrm{Li}$ et al., 2016). In fact, P300 activity varies in time-frequency amplitude and topography (Demiralp et al., 2001; Friedman et al., 2001). Brain activity in delta and theta bands is demonstrated to underlie P300 ERP (Başar-Eroglu and Demiralp, 2001; Harper et al., 2014; Bender et al., 2015). In order to investigate the change of brain activity during P300 task, the time-frequency analysis can be used (Friedman et al., 2001; Bernat et al., 2007).

In the brain, information is processed between specialized and spatially distributed but functionally linked regions (Bullmore and Sporns, 2009; Tian et al., 2013; Zhang et al., 2015; Bassett and Sporns, 2017; Li et al., 2018a). The endogenous processing in the brain, which functions on large-scale areas including frontal and parietal lobes (Bledowski et al., 2004; Polich, 2007) and their interactions (Zhang et al., 2014; Li et al., 2015, 2016), contributes to the generation of P300 (Donchin and Coles, 1988). Lesions of brain regions, such as temporal-parietal junction, resulted in P300 deficits, i.e., the decreased amplitude and prolonged latency (Yamaguchi and Knight, 1991; Verleger et al., 1994).

There is a debate that which aspect of cognitive process in the brain can be reflected by the P300 (Linden, 2005; Polich, 2007; Rutiku et al., 2015), decision (O'Connell et al., 2012), stimulusresponse links (Verleger et al., 2014), or attentional resources allocation (Putnam and Roth, 1990). In order to investigate this debate, the effects of stimulus characteristics on P300 were studied (Polich and Bondurant, 1997; Steiner et al., 2013). Despite the differences of P300 characteristics (e.g., amplitude and latency), no findings on brain networks were reported for this issue. In this study, we assumed that when distinct contexts were first presented, the brain network architectures with varied efficiencies were activated, and then contributed to the generation of P300. Taking a T as the reference, two types of stimulus sequences exist; one sequence is an $\mathrm{S}$ preceding a $\mathrm{T}$ (ST sequence), and another occurs when a $\mathrm{T}$ is presented in advance (TT sequence). We then investigated how the brain adapted dynamically to external stimuli, and probed the effects of different contexts on P300 by comparing the brain networks between different stimulus conditions.

\section{MATERIALS AND METHODS}

\section{Participants}

This experiment was approved by the Institution Research Ethics Board of University of Electronic Science and Technology of China. Twenty-two healthy graduate students (males, age of 22-27 years) were compensated financially to participate in P300 experiments, after providing their signed written informed consent. All participants were right-handed (self-reported), and had the normal or correct-to-normal vision. None of them had a history of substance abuse and a personal or family history of psychiatric or neurological disease.

\section{Experiment Design}

In this study, the $\mathrm{T}$ and $\mathrm{S}$ stimulus were expressed as a downward- and upward-oriented triangle with a thin cross in the center, respectively. For each stimulus, the edge length of the triangle was $4^{\circ}$ visual angle. The black color was set as background color, and the white color was set as triangle color. The line widths in triangles were $1 \mathrm{~mm}$, and the triangles were isosceles. Before task, all subjects were required to be relaxed, to focus attention on task without extensive body motion, and to avoid eyes blinking. Details of experimental protocols (Figure 1) were depicted as follows.

In this study, an eyes-closed resting-state with a duration of 4 min and three runs of P300 tasks were included. Between each two adjacency periods, a short break (i.e., $1 \mathrm{~min}$ ) was given to subjects. Each run consisted of 150 trials (120 standards and 30 targets) that were randomly presented. In each trial, a bold solid cross appeared as an alert to warn subjects to focus their attention; after $250 \mathrm{~ms}$, a thin solid cross appeared as a cue to inform subjects that either a $\mathrm{T}$ or $\mathrm{S}$ would appear very soon; after $500 \mathrm{~ms}$, a stimulus was presented for $500 \mathrm{~ms}$, and at the same time, subjects were required to count the number of $\mathrm{T}$ stimulus. After a break with the $1 \mathrm{~s}$ duration, next trial initiated. When each run ended, subjects would verbally speak out the counted number.

\section{Data Recording}

Rest and task electroencephalogram (EEG) data sets of $64 \mathrm{Ag} / \mathrm{AgCl}$ electrodes that were positioned according to international 10/20 system were recorded by using BrainVision 2.0.1 (Brain Products, Munich, Germany). The predefined parameters of online bandpass filtering was $0.01-100 \mathrm{~Hz}$, and sampling rate was $500 \mathrm{~Hz}$. During online recording, 


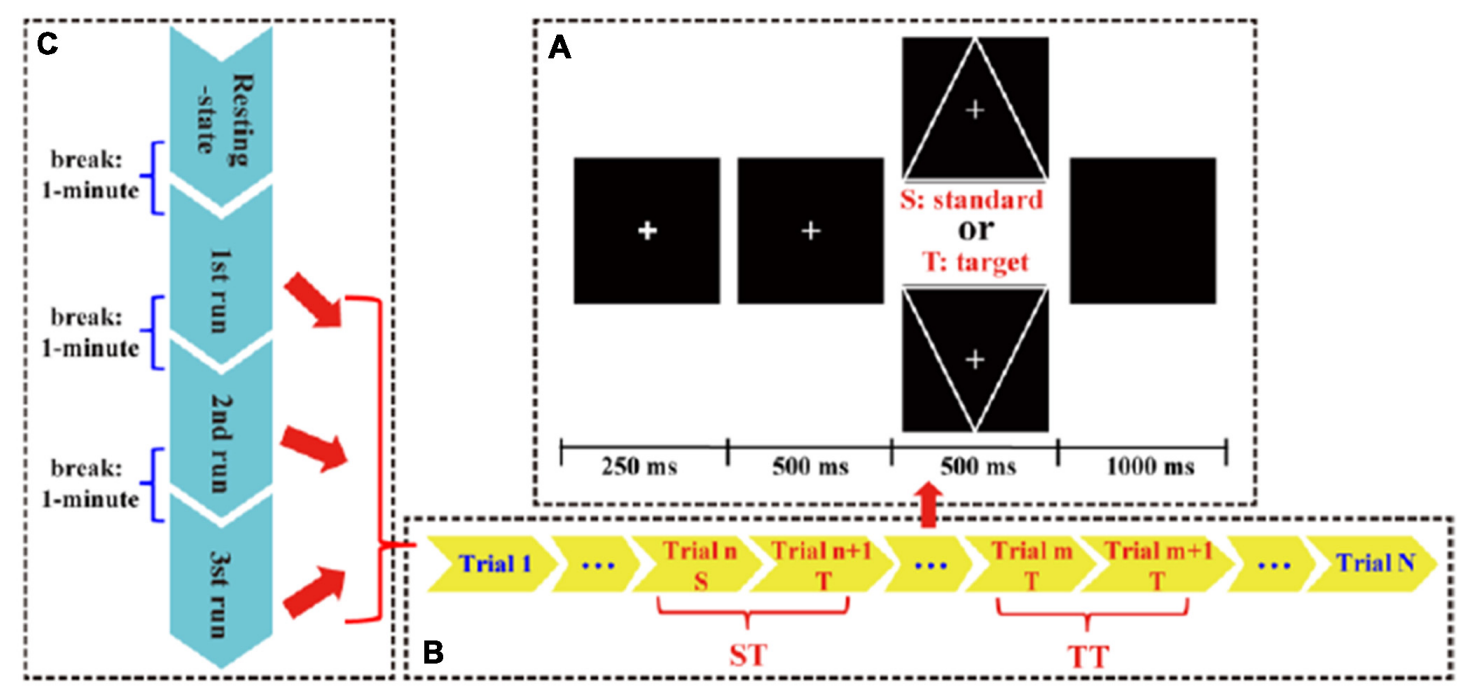

FIGURE 1 | The P300 protocol used in this study. (A) Each trial consisted of an alert of attention (250 ms), a cue of preparation (500 ms), a target (T) or standard (S) stimulus (500 ms), and a short black screen (1,000 ms). (B) Each run consisted of $150 \mathrm{~S} / \mathrm{T}$ stimuli. (C) Whole P300 task consisted of three runs.

electrodes $\mathrm{FCz}$ and $\mathrm{AFz}$ were regarded as reference and ground, respectively. Two additional electrodes, i.e., vertical (VEOG) and horizontal electrooculograms (HEOG), were used to monitor the eye movements. Particularly, VEOG was positioned at the right side of right eye, and HEOG was positioned below the left eye. During whole task, impedances for all electrodes were kept below $5 \mathrm{~K} \Omega$.

\section{Data Analyses}

In this study, three runs of task data sets were preprocessed with preprocessing procedures including, offline detecting and correcting blinks by using BrainAnalyzer 2.0.1, re-referencing to a neutral reference of Reference Electrode Standardization Technique (REST; Yao, 2001; Dong et al., 2017), 0.5-30 Hz bandpass filtering, $4.5 \mathrm{~s}$ data segmenting, and artifact-trial removal ( $\pm 75 \mu \mathrm{v}$ as threshold). Particularly, when performing data segmentation, we extracted two adjacent trials with the 2.25 s duration per trial, i.e., an S and a $\mathrm{T}$ of ST sequence and two T of TT sequence. Meanwhile, for artifact-trial removal, the $75 \mu \mathrm{v}$ threshold was used to automatically exclude artifacts with high amplitudes. If trial with largest absolute value of amplitude at any time point of any electrode exceeded $75 \mu \mathrm{v}$, this trial would be excluded from any further analysis. In this study, only two subjects experienced an exclusion of large trial number (ST: 11 and 16 trials, and TT: 6 and 5 trials). All trials $(17.08 \pm 3.30)$ in TT sequence were used in our analysis. Meanwhile, to acquire reliable result and to exclude the effect of trial number, based on the trial range of TT sequence, for ST sequence, we randomly selected the trial number within the same range for each subject, and $17.52 \pm 1.75$ trials in ST sequence were thereby included in our analysis. Thereafter, the trial numbers were then statistically investigated between two sequences by performing paired $t$-test, and no significant difference $(p=0.579)$ between two sequences was found.

\section{Time-Frequency Analysis}

Brain activity in low frequency range (i.e., delta and theta) is found to relate to the P300 (Harper et al., 2014; Bender et al., 2015). In this study, aiming to investigate the dynamic of task-related brain activity in frequency domain, we acquired the time-frequency distributions (TFDs) for two sequences by using the wavemenu of Matlab (v2014a). Particularly, we first calculated the TFDs for each artifact-free ST (also for TT) segment, and the TFDs were then averaged across segments to achieve TFDs mean of ST (also TT) sequence for each subject. Afterwards, to clearly illustrate the dynamic of brain activity, the TFDs mean was also grand-averaged across subjects.

\section{P300 Measurements}

One second interval of $[-200,800] \mathrm{ms}(0 \mathrm{~ms}$ represents the $\mathrm{T}$ onsets) was used to estimate the P300 amplitude. In this study, we averaged the $\mathrm{T}$ segments to achieve an averaged P300 ERP per condition for each subject. Afterwards, within time interval of [300 ms, $600 \mathrm{~ms}$ ] after T onsets, P300 amplitude on electrode $\mathrm{Pz}$ was calculated by averaging amplitudes within a time window of \pm 10 ms centered at largest positive peak. Meanwhile, we also estimated the corresponding P300 latency by obtaining the time point corresponding to the largest positive peak.

\section{Brain Networks}

The brain network is typically modeled by graph theory, and includes a collection of nodes and edges. In this study, 21 electrodes were set as network nodes, and the synchronized strengths between electrodes estimated by coherence were set as network edges. Coherence is usually used to measure the 
synchronized neuronal assembly at any given frequency bin $f$ between two signals, $x(t)$ and $y(t)$, and is formulated as,

$$
C_{x y}(f)=\left|R_{x y}(f)\right|^{2}=\frac{\left|P_{x y}(f)\right|^{2}}{P_{x x}(f) P_{y y}(f)}
$$

where $C_{x y}(f)$ and $R_{x y}(f)$ represent the coherence value and complex correlation coefficient between $x(t)$ and $y(t)$ at frequency $f$, respectively. $P_{x y}(f)$ represents the cross-spectrum of $x(t)$ and $y(t)$ at frequency $f$, and $P_{x x}(f)$ and $P_{y y}(f)$ represent the auto-spectrum at frequency $f$ of $x(t)$ and $y(t)$, respectively. These measurements of spectral densities were calculated from Fast Fourier Transform. For each frequency bin $f$, the $C_{x y}(f)$ is acquired by squaring the magnitude of the complex correlation coefficient $R$, which returns a real value within the range of $[0,1]$.

The short time series cannot exactly estimate the correlations between two electrodes, and may result in the spurious estimation of P300 network. In this study, EEG segments with relatively long interval (Chen et al., 2014; Diez et al., 2017), $2.25 \mathrm{~s}$ duration, were used to construct P300 networks. Meanwhile, P300 networks would be constructed in low frequency range, i.e., $1-10 \mathrm{~Hz}$ of our present study.

In this study, based on these artifact-free EEG segments, the coherence was first used to estimate the synchronized strength, $C_{x y}(f)$, between two electrodes at frequency $f$ per segment. Then, we averaged the synchronized strengths within the interested frequency range of $1-10 \mathrm{~Hz}$ to form an adjacency matrix per segment for each subject. Afterwards, for each subject, the final weighted EEG network that corresponded to either S or T of either ST or TT sequence, an adjacency matrix with dimension of $21 \times 21$, was obtained by averaging adjacency matrices across all artifact-free segments for each condition.

Network properties including clustering coefficients (CC) and characteristic path length (CPL) can be used to quantitatively measure brain network (Rubinov and Sporns, 2010). Theoretically, the CC indexes the functional segregation of a given network, and reflects the capacity for specialized processing to occur within densely interconnected regions. In contrast, the CPL is usually used to measure the corresponding functional integration, and indexes the ability to rapidly combine specialized information from distributed brain regions. In this study, we used the brain connectivity toolbox $\left(\mathrm{BCT}^{1}\right)$ to calculate the CC and CPL. Here, $w_{i j}$ represents the synchronized strength between nodes $i$ and $j$ estimated by coherence, $d_{i j}$ represents the shortest weighted path length, $N$ represents the number of all nodes, and $\Theta$ represents the set of network nodes. The CC and CPL were then formulized as follows:

$$
\begin{aligned}
C C & =\frac{1}{N} \sum_{i \in \Theta} \frac{\sum_{j, l \in \Theta}\left(w_{i j} w_{i l} w_{j l}\right)^{1 / 3}}{\sum_{j \in \Theta} w_{i j}\left(\sum_{j \in \Theta} w_{i j}-1\right)} \\
C P L & =\frac{1}{N} \sum_{i \in \Theta} \frac{\sum_{j \in \Theta, j \neq i} d_{i j}}{N-1}
\end{aligned}
$$

${ }^{1}$ http://www.nitrc.org/projects/bct/

\section{Statistical Analysis}

To investigate how the brain adjusted from last stimulus to adapt to a $\mathrm{T}$ stimulus, we then compared the EEG networks between two adjacency stimuli (i.e., $22 \times 21 \times 21$ of stimulus 1 vs. $22 \times 21 \times 21$ of stimulus 2$)$ in both sequences by using paired $t$-test, which resulted in a $21 \times 21$ matrix whose elements denoted significant $(p<0.05)$ or insignificant $(p>0.05)$ differences between two conditions. Particularly, if a network edge experienced significantly different between two conditions, this edge would be given an exact value (i.e., the quantitative difference of edge strengths between two stimuli); in contrast, if no significant difference was found, this edge would be discharged (i.e., giving a zero value). Thereafter, the $21 \times 21$ matrix was drawn on the scalp to visually display the topological differences between two stimuli, by using BCT function (i.e., "Brain_Graphic").

Afterwards, the paired $t$-test was also used to compare the P300 amplitude/latency and network properties between two stimuli to investigate the effect of stimulus sequence, as well as the differences of task activations between T in ST and T2 in TT. In this study, all comparisons were multiple corrected by performing false discovery rate (FDR) test.

\section{Differential Cortical Sources}

To investigate the brain regions that were important when responding to the second $\mathrm{T}$ in both sequences, we first estimated the standardized current densities that corresponded to trialaveraged ST/TT ERPs, by using standardized low-resolution electromagnetic tomography (sLORETA; Pascual-Marqui, 2002). The mean of sLORETA solutions within a time window of $\pm 10 \mathrm{~ms}$ centered at their own largest positive peak within 300-600 ms after stimulus onsets was then calculated and compared between two sequences. Considering that deep brain regions, such as parahippocampal gyrus, contributed little to scalp signals, and were demonstrated to have weak activations. In this study, these regions were also excluded from any further analysis.

In this study, the sLORETA employed the current density estimate given by minimum norm solution, and was capable of exact (zero-error) localization. In sLORETA, the solution space consists of 6,239 voxels (also includes hippocampi) at $5 \mathrm{~mm}$ spatial resolution in a 3 -shell realistic head model (Fuchs et al., 2002), using the digitized Montreal Neurological Institute atlas (152 template; Mazziotta et al., 2001).

\section{RESULTS}

Similar results could be found from both the TFDs (Figure 2) and scalp P300 ERP distributions (Figure 3). In Figure 2, when subjects responded to the presented $\mathrm{T}$ stimulus, the brain activity in low frequency range (i.e., range of $1-10 \mathrm{~Hz}$ ) could be found in both ST (Figure 2A) and TT (Figure 2B) sequences on electrode $\mathrm{Pz}$, respectively.

As illustrated in both Figures 2, 3, the obvious difference between two sequences was that, even though the TFDs in low 

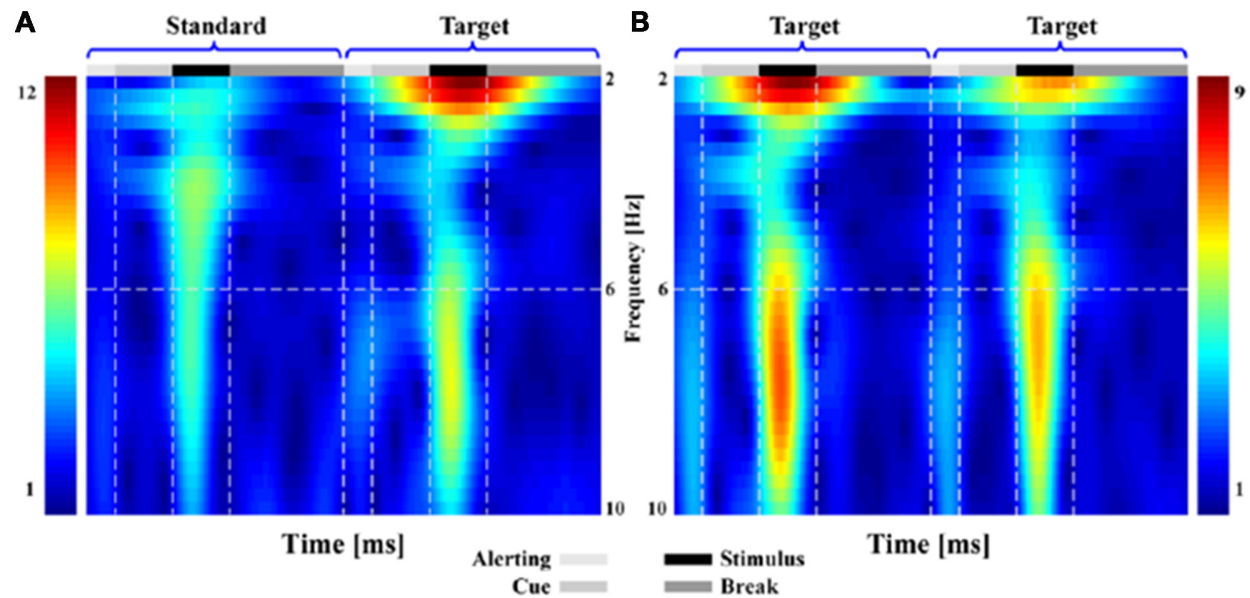

FIGURE 2 | The time-frequency distributions (TFDs) related to brain activity for both standard-target (ST) and target-to-target (TT) sequences during P300 tasks. Subfigure $(\mathbf{A}, \mathbf{B})$ denote the TFDs of ST and of TT on electrode Pz, respectively.

A

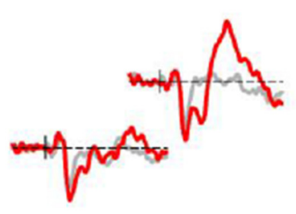

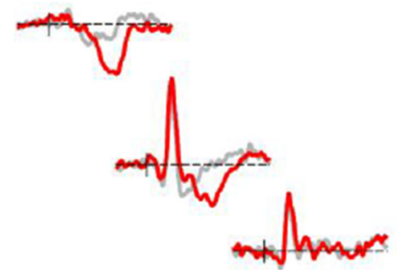

B
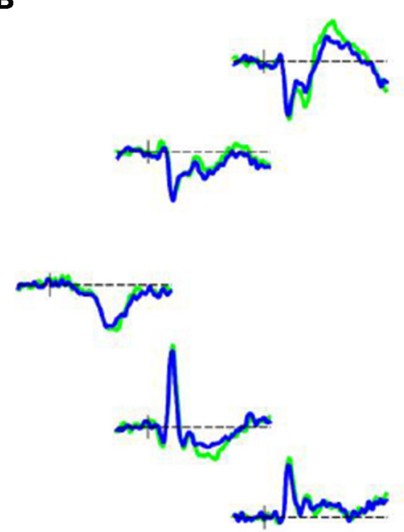

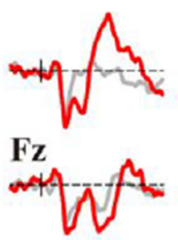<smiles>CCC1(C)CCCC1(C)C</smiles><smiles>CCCC1CCCC1CC</smiles>

$\mathrm{Cz}$

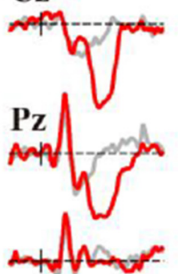

Sequence: ST

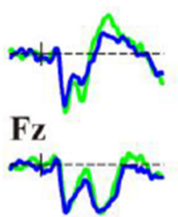

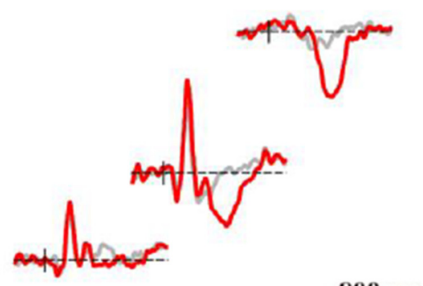



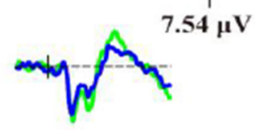

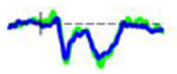

$\mathrm{Cz}$

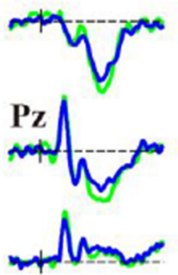

Sequence: TT

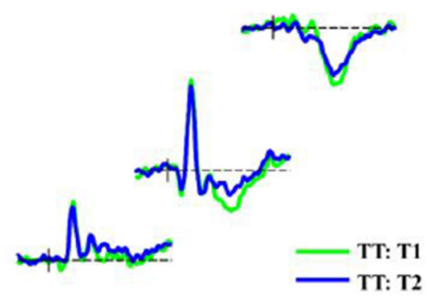

FIGURE 3 | The scalp distributions of averaged P300 event-related potentials (ERPs) for ST and TT sequences during P300 task. (A) P300 ERPs of ST.

(B) P300 ERPs of TT. In (A), the gray and red solid lines denote the waveforms evoked by the $S$ and T stimulus, respectively. In (B), T1 and T2 denote the first and second T stimulus in TT sequence, respectively; and the green and blue solid lines denote the waveforms evoked by the T1 and T2 stimulus, respectively. 


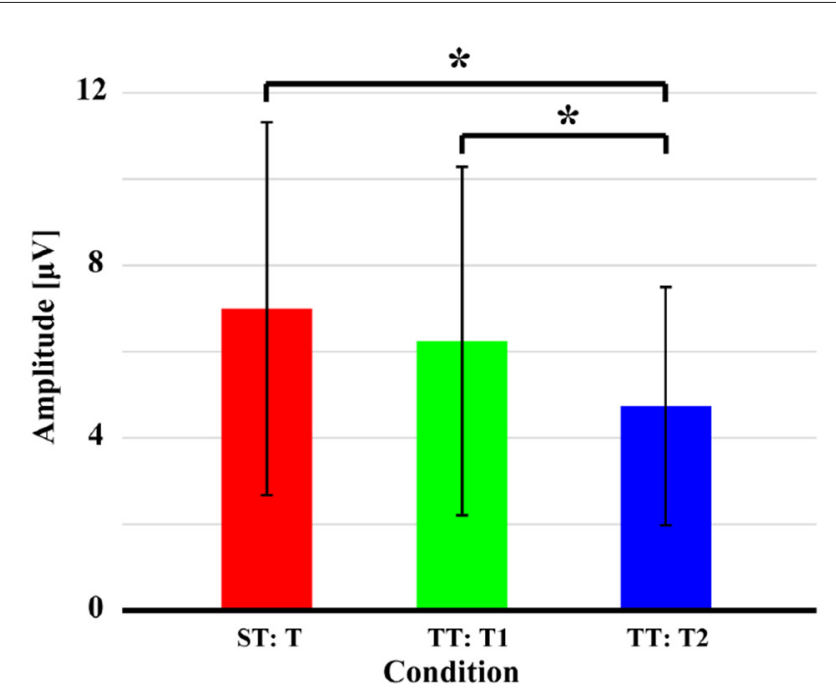

FIGURE 4 | P300 amplitudes evoked by the T in both ST and TT sequences. The black solid asterisks denote the significant $(p<0.05)$ differences of P300 amplitudes between two conditions. Error bars denote the standard deviations of P300 amplitudes among 22 subjects. Values are the means and standard deviations (Mean \pm SD) of P300 amplitudes.

frequency range could be found in TT sequence when both $\mathrm{T}$ stimuli were presented, the attenuated TFDs (Figure 2B) and P300 amplitudes (Figure 3B) were demonstrated for the second T stimulus (T2).

Figure 4 displays the quantitative differences of P300 amplitudes between two sequences. As demonstrated, no significant difference $(t=1.628, p=0.059, d f=21)$ of P300 amplitude between T in ST and T1 in TT was found; while significantly larger amplitude evoked by $\mathrm{T}$ in ST than that evoked by T2 in TT $(t=3.375, p=0.001, d f=21)$ and larger amplitude evoked by T1 than that evoked by T2 in TT $(t=2.963$, $p=0.004, d f=21$ ) were found. For latency, longer latency for T2 was demonstrated, compared to that of T1 in TT $(t=-1.467$, $p=0.079, d f=21)$ and T in ST $(t=-0.801, p=0.216, d f=21)$, although these were insignificant. Meanwhile, no difference of latency $(t=0.271, p=0.605, d f=21)$ between T in ST and T1 in TT was found.

Figure 5 shows the statistical differences of network topologies (Figure 5A) and network properties (Figures 5B,C) between two stimuli for both ST and TT sequences. Particularly, in Figure 5A, stronger network edges connecting frontal and parietal lobes were demonstrated for ST sequence $(p<0.05$, FDR correction), which was also evaluated by higher network efficiency (Figure 5B, CC: $t=-4.289, p=0.000, d f=21$; CPL: $t=4.114, p=0.000, d f=21$ ), when a $\mathrm{T}$ stimulus was presented after an S. However, for TT sequence, only smaller CC $(t=3.201, p=0.002, d f=21)$ and longer CPL $(t=-3.142$, $p=0.003, d f=21)$, which reflected the attenuated network efficiency (Figure 5C), were demonstrated when an identical $\mathrm{T}$ stimulus was presented after the last $\mathrm{T}$.

Figure 6 finally demonstrates the differences of network topology, properties, and task activations between T in ST and
T2 in TT. Attenuated network edges (Figure 6A, $p<0.05$, FDR correction) and properties [smaller CC $(t=3.573, p=0.000$, $d f=21)$ and longer CPL $(t=-3.532, p=0.001, d f=21)$, Figure 6B] were found in TT sequence. Meanwhile, Figure 6C illustrates the stronger ( $p<0.05$, FDR correction) task activity mainly in frontal, temporal, and occipital lobes, when a $\mathrm{T}$ stimulus was presented after an S. Concretely, brain regions in both hemispheres, which included middle frontal gyrus (BA10, $11)$, inferior frontal gyrus (BA10, 11), superior frontal gyrus (BA11), middle temporal gyrus (BA21, 38), superior temporal gyrus (BA38), middle occipital gyrus (BA18), and cuneus (BA18, 19), were found to be significantly different between two sequences.

\section{DISCUSSION}

In this study, subjects were requested to mentally count the number of T stimulus as quickly and correctly as possible, once they noticed the presentation of a $\mathrm{T}$ stimulus. In the brain, target information was processed in multiple procedures including stimuli perception, information integration, decision processing, and neuronal response, which was thought to be attributed to the interacted activity in the brain (Zhang et al., 2014; Li et al., 2015, 2016).

The sequence effect originated in part from the operation of immediate memory for the preceding stimulus train (Polich and Bondurant, 1997). As sequence unfolded, variation in P300 amplitude was induced due to an automatic concomitant shift in perceptual expectancy fostered by stimulus changes. The P300 indexed an updating process, a $\mathrm{T}$ stimulus after long interval produced great template updating to refresh the representation (Polich, 1990; Gonsalvez et al., 2007) and, thus, produced large P300 amplitude. The TFDs in Figure 2 and scalp P300 ERP distributions in Figure 3 showed the effect of stimulus sequence on P300. Although T stimulus could evoke a P300 in both ST and TT sequences, the context (i.e., S or $\mathrm{T}$ ) preceding an adjacency $\mathrm{T}$ was demonstrated to affect the generation of P300. Particularly, ST sequence evoked a larger P300 amplitude than TT sequence, which was also illustrated by the difference of P300 amplitude between different conditions in Figure 4.

Since no response was required for the $\mathrm{S}$ in ST sequence, brain resources were then allocated to react to the T. Therefore, the $\mathrm{T}$ stimulus experienced a stronger network topology (Figure 5A) that connected multiple regions including frontal and parietal lobes, compared to the $S$ one. In essence, network properties can detect functional integration and segregation of a given network (Rubinov and Sporns, 2010), and the larger CC and shorter CPL denote an increase of information processing efficiency during task. In this study, the larger CC and shorter CPL of ST sequence shown in Figure 5B thus quantitatively measured the increased efficiency when processing T-related information. However, if two identical $\mathrm{T}$ stimuli were presented, the targettarget perception reminded the brain to respond equally to both stimuli. In essence, a relatively long TTI (e.g., $6 \mathrm{~s}$ or longer) could eliminate the effect of target probability (also 


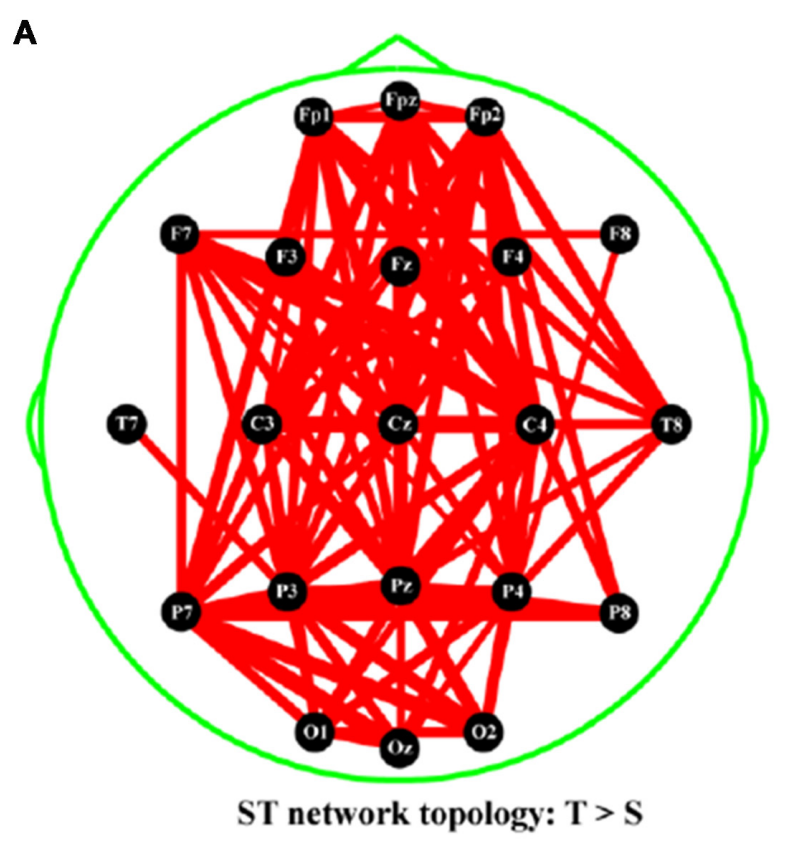

B

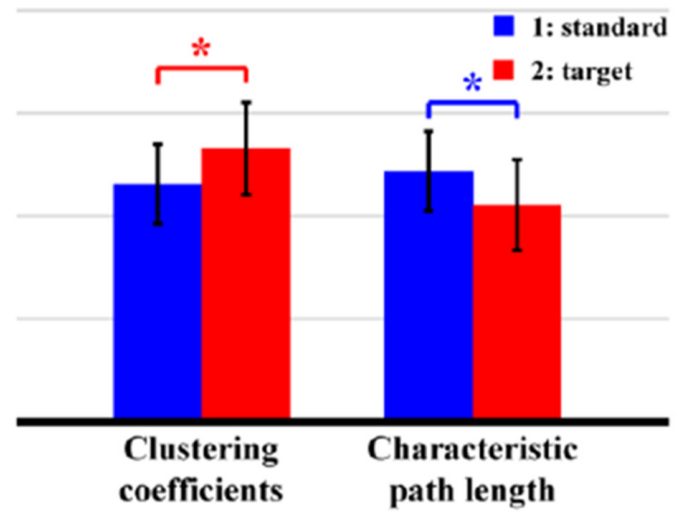

C

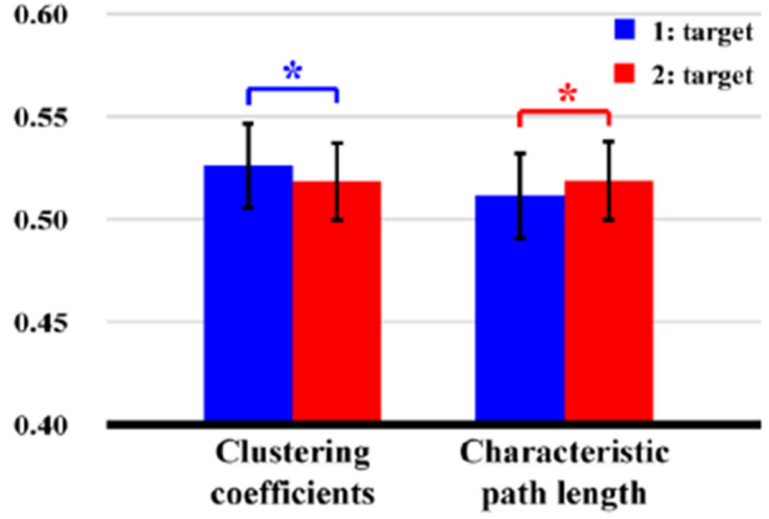

FIGURE 5 | Differences of network topologies and properties between two stimuli in both ST and TT sequences. (A) Network topology of ST sequence. (B,C) Network properties of ST and TT sequences, respectively. In (A), the red lines denote the stronger network edges of T than that of S stimulus; and the line widths denote the quantitative differences of edge strengths between two stimuli. In (B,C) the blue and red bars denote the network properties of first and second stimulus, respectively. ${ }^{*}$ Indicates $p<0.05$. Error bars denote the standard deviations of network properties among 22 subjects. Values are the means and standard deviations (Mean $\pm \mathrm{SD}$ ) of network properties.

stimulus sequence), and the P300 generation system could then recover fully from the last use (Polich, 1990; Polich and Bondurant, 1997). In our present study, the TT sequence produced a relatively short TTI of $2.25 \mathrm{~s}$. Although both P300 ERPs were evoked in TT sequence, due to short TTI, related brain resources could not fully and efficiently respond to the T2, which thus resulted in decreased P300 amplitude, prolonged P300 latency, and suppressed network properties (Figure 5C).

Once perceiving target information in the brain, related regions would be activated to be responsible for the corresponding dynamic processes, which included encoding, updating, and decaying of immediate memory trace (template) of the T stimulus (Owen et al., 1996; Polich and Criado, 2006; Luck et al., 2010). In this study, target information was first perceived and integrated in occipital lobe. Frontal lobe is demonstrated to be involved in multiple cognitive tasks and associated with effort and task difficulty (Paus et al., 1998). Here, the activation in frontal may reflect the general role of frontal lobe in response preparation and the monitoring of task information (Carter et al., 1998), which might relate to the early P300 process (i.e., command coding and decision processing; Li et al., 2009). Afterwards, the memory template of $\mathrm{T}$ stimulus was updated and transmitted to parietal lobe, a clear P300 component with large amplitude was thus evoked. As illustrated, the short interval affected the brain response to the 


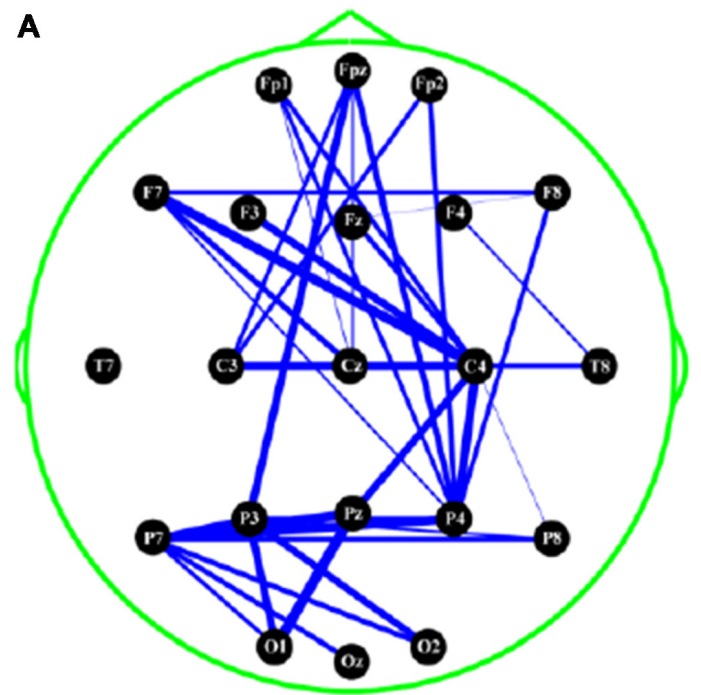

Network topology: T2 of TT $<$ T of ST
B



C
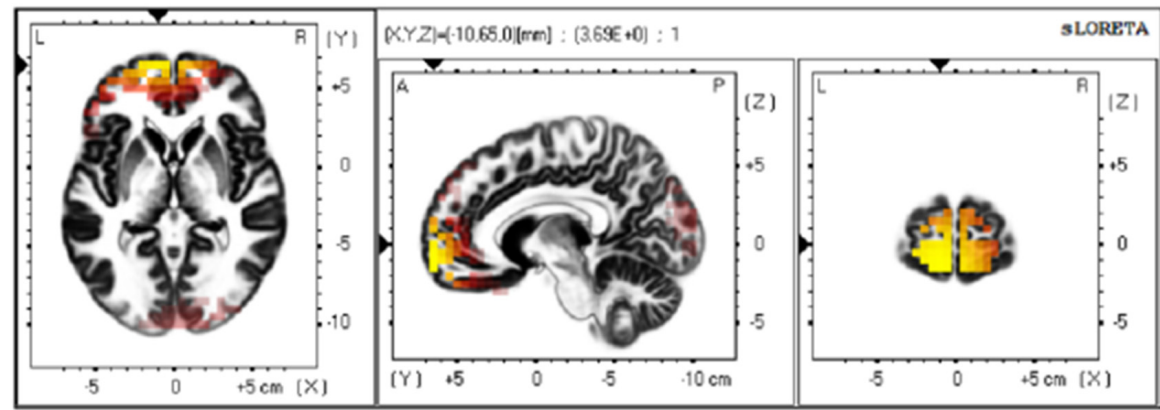

3.8

Task activation: T2 of TT $<$ T of ST

FIGURE 6 | Differences of network topology, properties, and task activation between T in ST and T2 in TT. (A) Network topology. (B) Network properties. (C) Task activation. In (A), the blue solid lines denote the weaker network edges of T2 in TT compared to that of T in ST; the line widths denote the quantitative differences of edge strengths between two conditions. In (B), the red and blue bars denote the network properties corresponding to T2 in TT and T in ST, respectively. ${ }^{*}$ Indicates $p<0.05$. Error bars denote the standard deviations of network properties among 22 subjects. Values are the means and standard deviations (Mean $\pm \mathrm{SD}$ ) of network properties.

T2 in TT sequence; we thus observed the stronger activations in multiple brain regions, such as middle frontal gyrus, superior frontal gyrus, and cuneus in Figure $6 \mathrm{C}$ for ST rather than TT sequence.

Findings of our study demonstrated the effect of stimulus sequence on brain network that subserved the target information processing in the brain. In the oddball task, ST sequence experienced an efficient allocation of brain resources, along with the high network efficiency, which would then promote the generation of P300 component with large amplitude. One possible limitation of this study was that subjects were all males, the gender difference might influence the findings to some degree, which was needed to be considered in the future work. Meanwhile, the investigation of single trial amplitude might be also helpful to deepen our understanding of this issue; in our future study, the combined single trial amplitude and brain network analysis could be considered, as well.

\section{AUTHOR CONTRIBUTIONS}

FL and PX conceived and designed the experiments and wrote the manuscript. FL performed the experiments. FL, CY, YJ, and YL analyzed the dataset. YS, JD, DY, and YZ provided some useful suggestions in manuscript writing.

\section{FUNDING}

This work was supported by the National Natural Science Foundation of China (\#61522105, \#61603344, \#81330032, \#\#1601136, and \#81771925), the Open Foundation of Henan Key Laboratory of Brain Science and Brain-Computer Interface Technology (No. HNBBL17001), the Longshan Academic Talent Research Supporting Program of SWUST (\#17LZX692), and ChengDu's HuiMin Projects of Science and Technology in 2013. 


\section{REFERENCES}

Başar-Eroglu, C., and Demiralp, T. (2001). Event-related theta oscillations: an integrative and comparative approach in the human and animal brain. Int. J. Psychophysiol. 39, 167-195. doi: 10.1016/s0167-8760(00)00140-9

Bassett, D. S., and Sporns, O. (2017). Network neuroscience. Nat. Neurosci. 20, 353-364. doi: 10.1038/nn.4502

Bender, S., Banaschewski, T., Roessner, V., Klein, C., Rietschel, M., Feige, B., et al. (2015). Variability of single trial brain activation predicts fluctuations in reaction time. Biol. Psychol. 106, 50-60. doi: 10.1016/j.biopsycho.2015. 01.013

Bernat, E. M., Malone, S. M., Williams, W. J., Patrick, C. J., and Iacono, W. G. (2007). Decomposing delta, theta, and $\alpha$ time-frequency ERP activity from a visual oddball task using PCA. Int. J. Psychophysiol. 64, 62-74. doi: 10.1016/j. ijpsycho.2006.07.015

Bledowski, C., Prvulovic, D., Hoechstetter, K., Scherg, M., Wibral, M., Goebel, R., et al. (2004). Localizing P300 generators in visual target and distractor processing: a combined event-related potential and functional magnetic resonance imaging study. J. Neurosci. 24, 9353-9360. doi: 10.1523/JNEUROSCI.1897-04.2004

Bullmore, E., and Sporns, O. (2009). Complex brain networks: graph theoretical analysis of structural and functional systems. Nat. Rev. Neurosci. 10, 186-198. doi: $10.1038 / \mathrm{nrn} 2575$

Busch, N. A., Schadow, J., Fründ, I., and Herrmann, C. S. (2006). Time-frequency analysis of target detection reveals an early interface between bottom-up and top-down processes in the $\gamma$-band. Neuroimage 29, 1106-1116. doi: 10.1016/j. neuroimage.2005.09.009

Carter, C. S., Braver, T. S., Barch, D. M., Botvinick, M. M., Noll, D., and Cohen, J. D. (1998). Anterior cingulate cortex, error detection, and the online monitoring of performance. Science 280, 747-749. doi: 10.1126/science.280. 5364.747

Chen, C. C., Syue, K. S., Li, K. C., and Yeh, S. C. (2014). Neuronal correlates of a virtual-reality-based passive sensory P300 network. PLoS One 9:e112228. doi: 10.1371/journal.pone.0112228

Demiralp, T., Ademoglu, A., Istefanopulos, Y., Başar-Eroglu, C., and Başar, E. (2001). Wavelet analysis of oddball P300. Int. J. Psychophysiol. 39, 221-227. doi: 10.1016/s0167-8760(00)00143-4

Diez, Á., Ranlund, S., Pinotsis, D., Calafato, S., Shaikh, M., Hall, M. H., et al. (2017). Abnormal frontoparietal synaptic gain mediating the P300 in patients with psychotic disorder and their unaffected relatives. Hum. Brain Mapp. 38, 3262-3276. doi: 10.1002/hbm.23588

Donchin, E., and Coles, M. G. H. (1988). Is the P300 component a manifestation of context updating? Behav. Brain Sci. 11, 357-374. doi: $10.1017 / \mathrm{s} 0140525 \times 00058027$

Dong, L., Li, F., Liu, Q., Wen, X., Lai, Y., Xu, P., et al. (2017). MATLAB toolboxes for reference electrode standardization technique (REST) of scalp EEG. Front. Neurosci. 11:601. doi: 10.3389/fnins.2017.00601

Duncan-Johnson, C. C., and Donchin, E. (1977). On quantifying surprise: the variation of event-related potentials with subjective probability. Psychophysiology 14, 456-467. doi: 10.1111/j.1469-8986.1977.tb01312.x

Farwell, L. A., and Donchin, E. (1988). Talking off the top of your head: toward a mental prosthesis utilizing event-related brain potentials. Electroenceph. Clin. Neurophysiol. 70, 510-523. doi: 10.1016/0013-4694(88)90149-6

Friedman, D., Cycowicz, Y. M., and Gaeta, H. (2001). The novelty P3: an event-related brain potential (ERP) sign of the brain's evaluation of novelty. Neurosci. Biobehav. Rev. 25, 355-373. doi: 10.1016/s0149-7634(01) 00019-7

Fuchs, M., Kastner, J., Wagner, M., Hawes, S., and Ebersole, J. S. (2002). A standardized boundary element method volume conductor model. Clin. Neurophysiol. 113, 702-712. doi: 10.1016/s1388-2457(02)00030-5

Gonsalvez, C. J., Barry, R. J., Rushby, J. A., and Polich, J. (2007). Target-totarget interval, intensity, and P300 from an auditory single-stimulus task. Psychophysiology 44, 245-250. doi: 10.1111/j.1469-8986.2007.00495.x

Harper, J., Malone, S. M., and Bernat, E. M. (2014). Theta and delta band activity explain N2 and P3 ERP component activity in a go/no-go task. Clin. Neurophysiol. 125, 124-132. doi: 10.1016/j.clinph.2013.06.025

Howe, A. S., Bani-Fatemi, A., and De Luca, V. (2014). The clinical utility of the auditory P300 latency subcomponent event-related potential in preclinical diagnosis of patients with mild cognitive impairment and Alzheimer's disease. Brain Cogn. 86, 64-74. doi: 10.1016/j.bandc.2014.01.015

Li, F., Chen, B., Li, H., Zhang, T., Wang, F., Jiang, Y., et al. (2016). The time-varying networks in P300: a task-evoked EEG study. IEEE Trans. Neural Syst. Rehabil. Eng. 24, 725-733. doi: 10.1109/TNSRE.2016. 2523678

Li, F., Liu, T., Wang, F., Li, H., Gong, D., Zhang, R., et al. (2015). Relationships between the resting-state network and the P3: evidence from a scalp EEG study. Sci. Rep. 5:15129. doi: 10.1038/srep15129

Li, F., Peng, W., Jiang, Y., Song, L., Liao, Y., Yi, C., et al. (2018a). The dynamic brain networks of motor imagery: time-varying causality analysis of scalp EEG. Int. J. Neural Syst. doi: 10.1142/S0129065718500168 [Epub ahead of print].

Li, F., Wang, J., Jiang, Y., Si, Y., Peng, W., Song, L., et al. (2018b). Top-down disconnectivity in schizophrenia during P300 tasks. Front. Comput. Neurosci. 12:33. doi: $10.3389 /$ fncom. 2018.00033

Li, Y., Pan, J., Wang, F., and Yu, Z. (2013). A hybrid BCI system combining P300 and SSVEP and its application to wheelchair control. IEEE Trans. Biomed. Eng. 60, 3156-3166. doi: 10.1109/TBME.2013.2270283

Li, Y., Wang, L. Q., and Hu, Y. (2009). Localizing P300 generators in high-density event-related potential with fMRI. Med. Sci. Monit. 15, MT47-MT53.

Linden, D. E. (2005). The P300: where in the brain is it produced and what does it tell us? Neuroscientist 11, 563-576. doi: 10.1177/10738584052 80524

Luck, D., Danion, J. M., Marrer, C., Pham, B. T., Gounot, D., and Foucher, J. (2010). The right parahippocampal gyrus contributes to the formation and maintenance of bound information in working memory. Brain Cogn. 72, 255-263. doi: 10.1016/j.bandc.2009.09.009

Mazziotta, J., Toga, A., Evans, A., Fox, P., Lancaster, J., Zilles, K., et al. (2001). A probabilistic atlas and reference system for the human brain: International Consortium for Brain Mapping (ICBM). Philos. Trans. R. Soc. Lond. B Biol. Sci. 356, 1293-1322. doi: 10.1098/rstb.2001.0915

O'Connell, R. G., Dockree, P. M., and Kelly, S. P. (2012). A supramodal accumulation-to-bound signal that determines perceptual decisions in humans. Nat. Neurosci. 15, 1729-1735. doi: 10.1038/nn.3248

Owen, A. M., Milner, B., Petrides, M., and Evans, A. C. (1996). A specific role for the right parahippocampal gyrus in the retrieval of object-location: a positron emission tomography study. J. Cogn. Neurosci. 8, 588-602. doi: 10.1162/jocn. 1996.8.6.588

Pan, J., Xie, Q., He, Y., Wang, F., Di, H., Laureys, S., et al. (2014). Detecting awareness in patients with disorders of consciousness using a hybrid brain-computer interface. J. Neural. Eng. 11:056007. doi: 10.1088/17412560/11/5/056007

Pascual-Marqui, R. D. (2002). Standardized low-resolution brain electromagnetic tomography (sLORETA): technical details. Methods Find. Exp. Clin. Pharmacol. 24, 5-12.

Pashler, H. (1994). Dual-task interference in simple tasks: data and theory. Psychol. Bull. 116, 220-244. doi: 10.1037/0033-2909.116.2.220

Paus, T., Koski, L., Caramanos, Z., and Westbury, C. (1998). Regional differences in the effects of task difficulty and motor output on blood flow response in the human anterior cingulate cortex: a review of 107 PET activation studies. Neuroreport 9, R37-R47. doi: 10.1097/00001756-19980622000001

Pokorny, C., Klobassa, D. S., Pichler, G., Erlbeck, H., Real, R. G., Kübler, A., et al. (2013). The auditory P300-based single-switch brain-computer interface: paradigm transition from healthy subjects to minimally conscious patients. Artif. Intell. Med. 59, 81-90. doi: 10.1016/j.artmed.2013. 07.003

Polich, J. (1990). P300, probability, and interstimulus interval. Psychophysiology 27, 396-403. doi: 10.1111/j.1469-8986.1990.tb02333.x

Polich, J. (2007). Updating P300: an integrative theory of P3a and P3b. Clin. Neurophysiol. 118, 2128-2148. doi: 10.1016/j.clinph.2007.04.019

Polich, J., and Bondurant, T. (1997). P300 sequence effects, probability, and interstimulus interval. Physiol. Behav. 61, 843-849. doi: 10.1016/s00319384(96)00564-1

Polich, J., and Criado, J. R. (2006). Neuropsychology and neuropharmacology of P3a and P3b. Int. J. Psychophysiol. 60, 172-185. doi: 10.1016/j.ijpsycho.2005. 12.012 
Putnam, L. E., and Roth, W. T. (1990). Effects of stimulus repetition, duration, and rise time on startle blink and automatically elicited P300. Psychophysiology 27, 275-297. doi: 10.1111/j.1469-8986.1990.tb00383.x

Risetti, M., Formisano, R., Toppi, J., Quitadamo, L. R., Bianchi, L., Astolfi, L., et al. (2013). On ERPs detection in disorders of consciousness rehabilitation. Front. Hum. Neurosci. 7:775. doi: 10.3389/fnhum.2013.00775

Rubinov, M., and Sporns, O. (2010). Complex network measures of brain connectivity: uses and interpretations. Neuroimage 52, 1059-1069. doi: 10.1016/j.neuroimage.2009.10.003

Rutiku, R., Martin, M., Bachmann, T., and Aru, J. (2015). Does the P300 reflect conscious perception or its consequences? Neuroscience 298, 180-189. doi: 10.1016/j.neuroscience.2015.04.029

Squires, K. C., Wickens, C., Squires, N. K., and Donchin, E. (1976). The effect of stimulus sequence on the waveform of the cortical event-related potential. Science 193, 1142-1146. doi: 10.1126/science.959831

Squires, N. K., Squires, K. C., and Hillyard, S. A. (1975). Two varieties of long-latency positive waves evoked by unpredictable auditory stimuli in man. Electroenceph. Clin. Neurophysiol. 38, 387-401. doi: 10.1016/00134694(75)90263-1

Steiner, G. Z., Brennan, M. L., Gonsalvez, C. J., and Barry, R. J. (2013). Comparing P300 modulations: target-to-target interval versus infrequent nontarget-tonontarget interval in a three-stimulus task. Psychophysiology 50, 187-194. doi: 10.1111/j.1469-8986.2012.01491.x

Stevens, M. C., Calhoun, V. D., and Kiehl, K. A. (2005). fMRI in an oddball task: effects of target-to-target interval. Psychophysiology 42, 636-642. doi: 10.1111/j. 1469-8986.2005.00368.x

Tian, Y., Ma, W., Tian, C., Xu, P., and Yao, D. (2013). Brain oscillations and electroencephalography scalp networks during tempo perception. Neurosci. Bull. 29, 731-736. doi: 10.1007/s12264-013-1352-9

Turetsky, B. I., Dress, E. M., Braff, D. L., Calkins, M. E., Green, M. F., Greenwood, T. A., et al. (2015). The utility of P300 as a schizophrenia endophenotype and predictive biomarker: clinical and socio-demographic modulators in COGS-2. Schizophr. Res. 163, 53-62. doi: 10.1016/j.schres.2014. 09.024

van Dinteren, R., Arns, M., Jongsma, M. L., and Kessels, R. P. (2014). Combined frontal and parietal P300 amplitudes indicate compensated cognitive processing across the lifespan. Front. Aging Neurosci. 6:294. doi: 10.3389/fnagi.2014.00294

Verleger, R. (1987). Sequential effects on P3 in a counting task: a partial replication. Biol. Psychol. 25, 221-246. doi: 10.1016/0301-0511(87)90049-4
Verleger, R., Baur, N., Metzner, M. F., and Smigasiewicz, K. (2014). The hard oddball: effects of difficult response selection on stimulus-related P3 and on response-related negative potentials. Psychophysiology 51, 1089-1100. doi: 10.1111 /psyp. 12262

Verleger, R., Heide, W., Butt, C., and Kömpf, D. (1994). Reduction of P3b in patients with temporo-parietal lesions. Cogn. Brain Res. 2, 103-116. doi: 10.1016/0926-6410(94)90007-8

Wang, L., Zheng, J., Huang, S., and Sun, H. (2015). P300 and decision making under risk and ambiguity. Comput. Intell. Neurosci. 2015:108417. doi: 10.1155/2015/108417

Yamaguchi, S., and Knight, R. T. (1991). Anterior and posterior association cortex contributions to the somatosensory-P300. J. Neurosci. 11, 2039-2054. doi: 10.1523/JNEUROSCI.11-07-02039.1991

Yao, D. (2001). A method to standardize a reference of scalp EEG recordings to a point at infinity. Physiol. Meas. 22, 693-711. doi: 10.1088/0967-3334/22/ 4/305

Yin, E., Zeyl, T., Saab, R., Hu, D., Zhou, Z., and Chau, T. (2016). An auditory-tactile visual saccade-independent P300 brain-computer interface. Int. J. Neural Syst. 26:1650001. doi: 10.1142/S0129065716500015

Yin, E., Zhou, Z., Jiang, J., Chen, F., Liu, Y., and Hu, D. (2013). A novel hybrid BCI speller based on the incorporation of SSVEP into the P300 paradigm. J. Neural Eng. 10:026012. doi: 10.1088/1741-2560/10/2/026012

Zhang, Y., Guo, D., Cheng, K., Yao, D., and Xu, P. (2015). The graph theoretical analysis of the SSVEP harmonic response networks. Cogn. Neurodyn. 9, 305-315. doi: 10.1007/s11571-015-9327-3

Zhang, Y., Tang, A. C., and Zhou, X. (2014). Synchronized network activity as the origin of a P300 component in a facial attractiveness judgment task. Psychophysiology 51, 285-289. doi: 10.1111/psyp.12153

Conflict of Interest Statement: The authors declare that the research was conducted in the absence of any commercial or financial relationships that could be construed as a potential conflict of interest.

Copyright (c) 2019 Li, Yi, Jiang, Liao, Si, Dai, Yao, Zhang and Xu. This is an open-access article distributed under the terms of the Creative Commons Attribution License (CC BY). The use, distribution or reproduction in other forums is permitted, provided the original author(s) and the copyright owner(s) are credited and that the original publication in this journal is cited, in accordance with accepted academic practice. No use, distribution or reproduction is permitted which does not comply with these terms. 